\title{
Mochila y carro escolar: análisis cinemático usando distintas cargas
}

\section{Premio Congreso SIBB 2016}

\author{
E. ORANTES-GONZÁLEZ, J. HEREDIA-JIMÉNEZ \\ Departamento Educación Física y Deportiva, Universidad de Granada
}

\begin{abstract}
Resumen
Introducción. El uso de los carros escolares es cada vez mayor en niños de educación primaria. Para clarificar las recomendaciones entre carro y/o mochila, en este estudio se ha realizado un análisis cinemático transportando distintas cargas con ambas opciones de transporte.

Métodos. 14 sujetos fueron evaluados (9.6+1.8 años). Un sistema de captura de movimiento $3 D$ fue utilizado para analizar la locomoción caminando sin carga, llevando una mochila y tirando del carro, ambas con el 10\%, 15\% y 20\% peso corporal (BW) del sujeto. Los parámetros cinemáticos del tronco, pelvis, cadera y rodilla fueron analizados.

Resultados. El tronco aumentó la flexión conforme aumentaba el peso del carro y la mochila. La pelvis y cadera aumentaron su flexión caminando con la mochila cargada comparado con caminar sin carga. La cadera aumentó su aducción usando la mochila con el $10 \%$ y el $20 \%$ BW con respecto al uso del carro, y disminuyó los movimientos de rotación $(20 \% \mathrm{BW})$. La rodilla no mostró diferencias en ninguno de los planos, tampoco la pelvis ni el tronco en los planos frontal y lateral.

Conclusiones. El uso del carro escolar requiere menos adaptaciones que la mochila con las mismas cargas y un comportamiento postural más próximo a caminar sin carga.
\end{abstract}

Palabras clave: Mochila de ruedas, cinemática, estudiantes, carga.

\begin{abstract}
Introduction. The use of school trolleys has increased in Elementary school students. To clarify recommendations about the use of trolleys and backpacks, this study compared the effects of carrying a backpack and pulling a trolley with different loads on gait kinematics.

Method. Fourteen students were evaluated $(9.6+1.8$ years). A 3D motion capture system was used to capture gait in the following conditions: walking without a school bag, carrying a backpack and pulling a trolley with 10\%, 15\% and $20 \%$ of their body weight $(B W)$. 3D kinematics of the thorax, pelvis, hip and knee were computed.

Results. Thorax flexion increased as backpack and trolley loads were higher. The hip and the pelvis increased flexion while walking with a backpack compared to a no-load condition. The hip also increased adduction while carrying a backpack loaded with $10 \%$ and $20 \%$ BW compared to trolley conditions. Rotation movements decreased in the hip while carrying a backpack with $20 \% \mathrm{BW}$. The pelvis and the thorax did not show significant differences in frontal and lateral planes, while the knee did not show differences in any of the three planes analyzed.

Conclusions. Pulling a trolley resulted in shorter kinematic adaptations than carrying a backpack with the same loads and also, the gait was more similar to the baseline gait.
\end{abstract}

Keywords: Wheeled backpack, kinematic, students, load.

Correspondencia:

Eva Orantes González. Departamento Educación Física y Deportiva, Universidad de Granada,

Carretera de Alfacar s/n, Granada, 18011, España.

Email: maevor@ugr.es 


\section{Introducción}

Los escolares diariamente usan una mochila o un carro escolar para transportar los libros y otros materiales escolares. Las recomendaciones en cuánto a peso de la mochila escolar oscilan entre el 10-20\% del peso corporal (BW) del niño [1-3]. Aunque estas recomendaciones no son siempre respetadas tal y como han mostrado estudios previos: entre el $40 \%$ y el $72 \%$ de los escolares suelen cargar mochilas más pesadas del 15\% BW [4-7].

Ante esta situación, el carro escolar surge como una alternativa a la mochila tradicional.

En estudios realizados anteriores a 2014 con muestra española, el carro era la opción elegida por entre el $31 \%$ y el $44 \%$ de los escolares [8-10]. En un estudio reciente se ha obtenido que los escolares usuarios de carro escolar suponen un $53.8 \%$ frente a los que prefieren usar la mochila, por lo tanto, el carro se muestra como la opción más utilizada en el rango de edad de 6 a 12 años [6].

Las ruedas que lleva implementadas el carro evita que la carga a transportar recaiga sobre la espalda de los escolares, como ocurre en el caso de la mochila. Esto podría generar una percepción de seguridad y cuidado de la espalda que hace que el $57 \%$ de los padres de los escolares consideren el carro como una opción que requiere menos esfuerzo y les resulta más cómodo para sus hijos [8]. En contra, el uso del carro escolar conlleva un esfuerzo asimétrico. Además los usuarios de carro transportan entre un $6 \%$ y un $30 \%$ más peso que la mochila [6,7]. Probablemente esta diferencia de peso se deba a la propia estructura del carro con las ruedas, que lo haga de por sí, más pesado que la mochila. Por otro lado, cuándo debemos salvar un obstáculo con el carro, como por ejemplo son las escaleras, conlleva un incremento de la carga asimétrica sobre el escolar al transportarlo sobre un solo hombro [11].

En relación al análisis de los aspectos posturales, la mayoría de estudios han obtenido que transportar una mochila cargada con un peso de entre el $10-20 \%$ BW produce principalmente un aumento de la flexión de los segmentos proximales (tronco y pelvis) para compensar la carga que es soportada sobre la espalda [12-17]. En los segmentos distales (tobillo y rodilla) los cambios producidos como consecuencia de transportar una mochila cargada han estado menos marcados que los producidos en los segmentos proximales [18]. En el caso del carro, su uso ha estado relacionado con un aumento de la flexión de la cadera y de la pelvis cuando se transporta el 20\% BW [19]. Además, el carro escolar se ha considerado una buena opción para los escolares cuando su peso oscila entre el 10-15\% BW [19].

Otros estudios previos también han analizado por separado los efectos de transportar un carro o una mochila. De esta forma, Schmidt y Docherty [20] usando un diseño entregrupo, recomendaron hacer mayor hincapié en el uso de la mochila con una carga recomendada en lugar de usar un carro escolar. Por el contrario, en un reciente estudio se concluyó que el uso del carro escolar con el 15\% BW puede ser considerado una opción segura para los escolares $[17,19]$. Dada la importante presencia que supone el uso del carro escolar, este estudio pretende analizar las principales adaptaciones posturales que se producen llevando un carro y una mochila con distintas cargas.

\section{Materiales y Métodos}

\section{Participantes}

Catorce escolares de educación primaria ( 8 niñas y 6 niños) fueron analizados. La media de edad obtenida fue $9.6 \pm 1.8$ años, la altura media $1.5 \pm 0.1 \mathrm{~m}$ y el peso medio $39.9 \pm 12.3$ $\mathrm{kg}$. Todos ellos participaron de forma voluntaria en el estudio. Además el 50\% de ellos usaban diariamente el carro, y el otro 50\% prefería usar la mochila. Como criterios de exclusión se consideró padecer cualquier problema traumatológico o neurológico que impidiera transportar caminando una mochila o un carro escolar. Los padres/madres o tutores legales de los sujetos firmaron un consentimiento informado. El Comité de Ética de la Universidad de Granada aprobó este estudio.

\section{Protocolo}

En primer lugar, los sujetos fueron me- 
didos y pesados en un tallímetro y báscula (SECA769, Hamburgo, Alemania). Una vez completada esta parte, se procedió a la colocación de los marcadores reflectantes (figura 1) siguiendo el modelo CAST y que ha sido validado en un estudio previo [21]. Los marcadores epidérmicos fueron colocados según se detalla en la figura 1.

Un sistema de captura del movimiento 3D con 9 cámaras grabando a $250 \mathrm{~Hz}$ (Qualisys AB, Göteborg, Suecia) fue colocado en la zona central de un pasillo de locomoción de 15 metros para evitar la fase de aceleración y desaceleración de los sujetos en cada una de las condiciones. Tras una fase de familiarización, cada uno de los sujetos caminaron du- rante 1 minuto en las siguientes condiciones experimentales: caminando sin mochila ni carro (considerada como condición "control"), llevando una mochila con las 2 asas sobre la espalda y tirando de un carro escolar, ambos con las siguientes cargas: $10 \%, 15 \%$ y $20 \%$ BW. Las condiciones fueron aleatorizadas y se consideró 3 minutos de descanso entre cada condición para evitar la aparición de fatiga.

La mochila se colocó sobre los dos hombros y con una posición centrada en la espalda. En el caso del carro, los sujetos utilizaban la mano dominante para tirar del mismo (en todos casos fue la derecha). Para conseguir el porcentaje de carga pretendido, se utilizaron libros con distintos pesos, colocando los

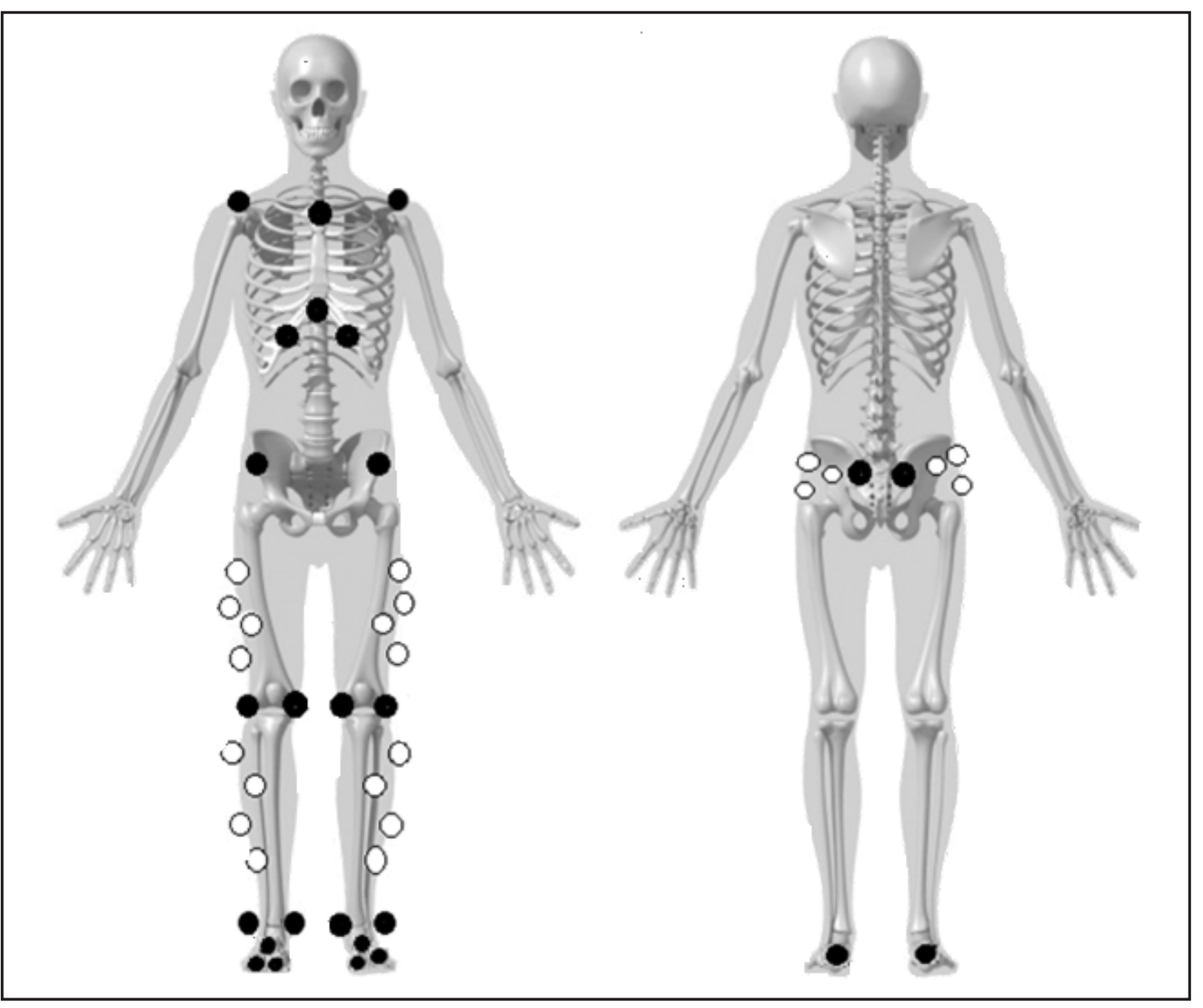

Figura 1. Representación del modelo de marcadores utilizado. Marcadores blancos: clusters utilizados. Marcadores negros: marcadores situados en los puntos acromiocraviculares, punto medio interclavicular, punto inferior del esternón, cartílago costal $7^{\mathrm{a}}$ costilla, espina iliaca superior anterior y posterior, epicóndilo femoral medial y lateral, maléolo medial y lateral, $1^{\mathrm{a}}$ y $5^{\mathrm{a}}$ cabeza del metatarso, $2^{\mathrm{a}}$ articulación metatarso-falángica y prominencia mayor posterior del calcáneo. Figura adaptada de "Marker Set Guidelines", C-Motion's Visual3D biomechanics research software. 
más pesados siempre en la zona interna de la mochila/carro. El software Visual 3D v.5 (CMotion Inc., Germantown, EE.UU.) fue utilizado para obtener las variables cinemáticas de la locomoción en cada una de las condiciones.

\section{Variables analizadas}

Se obtuvieron la media y desviación típica medida en grados de los movimientos en el plano sagital (flexión-extensión), plano frontal (aducción-abducción) y plano transversal (rotación interna-externa), de los siguientes segmentos corporales: tronco, cadera, pelvis y rodilla. El tronco fue analizado como el ángulo formado por el segmento tórax y la cadera, la cadera como el ángulo formado por la pelvis y el fémur, la pelvis fue analizada con respecto al sistema de referencia global del laboratorio, y finalmente la rodilla, que fue analizada como el ángulo formado por el fémur y la tibia. Todas las variables fueron relativizadas considerando un ciclo de marcha como el 100\%, y se usó la media de los ciclos derechos e izquierdos registrados.

\section{Análisis estadístico}

El software SPSS v.23 (SPSS Inc, Chicago, Illinois) fue utilizado para el análisis de los datos. Se realizó un análisis de la normalidad usando el test de Shapiro-Wilks, y se midió la esfericidad de los datos mediante el test de Mauchly. Para comparar las distintas condiciones de carga, un ANOVA de medidas repetidas fue aplicado. En todos los test aplicados, el nivel de significación fue $\mathrm{p}<0.05$.

\section{Resultados}

Los movimientos obtenidos en el plano sagital del tronco, pelvis y cadera se muestran en la Figura 2.

En el plano frontal solo la cadera mostró un aumento significativo de su aducción entre la condición mochila 10\% BW comparado con el carro $10 \%$ BW ( $<<0.05)$, y también entre mochila 20\% BW y carro $20 \%$ BW $(\mathrm{p}<0.05)$. En el plano transversal, la cadera fue nuevamente la única afectada por las distintas condiciones de carga, obteniendo una disminución significativa de la rotación comparando la condición control con el 20\% BW usando mochila $(\mathrm{p}<0.05)$ (Tabla 1). La rodilla no mostró diferencias significativas en ninguno de los planos analizados (Tabla 1).

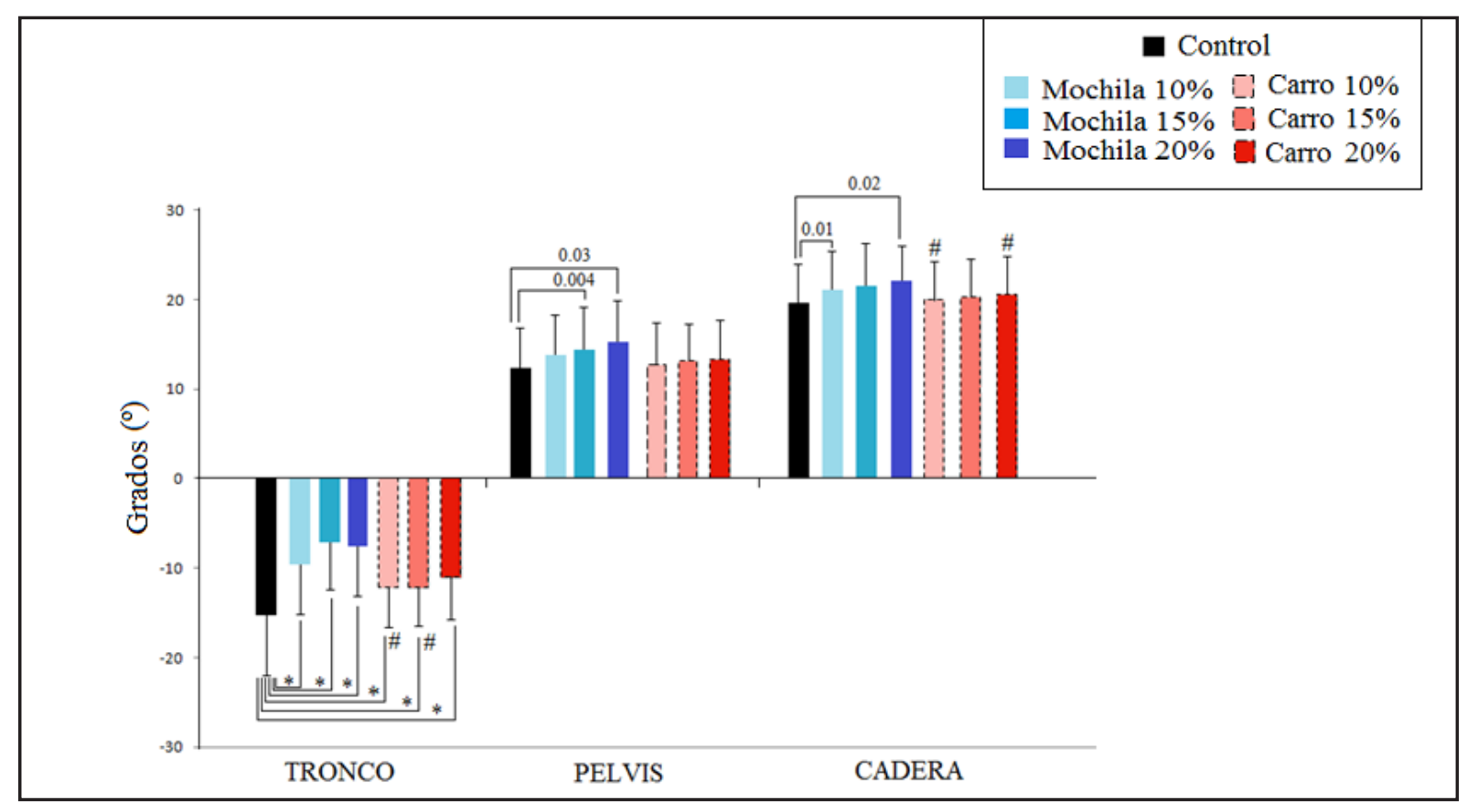

Figura 2. Movimientos en el plano sagital del tronco, pelvis y cadera. Valores positivos indican flexión, valores negativos extensión. *: $\mathrm{p}<0.001$; \#: $\mathrm{p}<0.05$ comparando mochila-carro con la misma carga. 


\section{Discusión}

En este estudio se han analizado las principales adaptaciones posturales del tronco, cadera, pelvis y rodilla utilizando las dos formas de transporte de material escolar preferido por los estudiantes: usando una mochila y usando un carro escolar. Además se han analizado las cargas usadas por la mayor parte de estudios científicos previos relacionados con el tema: $10 \%, 15 \%$ y $20 \%$ BW del sujeto [13,22-24].
Una de las principales adaptaciones obtenidas en este estudio ha sido el aumento de la flexión del tronco, tanto llevando la mochila con las distintas cargas, como usando el carro. Esta flexión del tronco ha estado previamente relacionada con una adaptación para compensar el peso transportado sobre la espalda usando la mochila [24-26] y también con el uso del carro $[17,19]$. La menor flexión del tronco necesaria cuando se utilizó el carro en comparación con la mochila, puede deberse al hecho de

\begin{tabular}{|c|c|c|c|c|c|c|c|}
\hline \multirow{3}{*}{$\begin{array}{c}\text { Parámetros' } \\
\text { cinemáticos } \\
\text { (grados) }\end{array}$} & \multirow{3}{*}{ Control } & \multicolumn{6}{|c|}{ Condiciones experimentales } \\
\hline & & \multicolumn{3}{|c|}{ Mochila } & \multicolumn{3}{|c|}{ Carro escolar } \\
\hline & & $10 \%$ & $15 \%$ & $20 \%$ & $10 \%$ & $15 \%$ & $20 \%$ \\
\hline \multicolumn{8}{|l|}{ Tronco } \\
\hline $\begin{array}{l}\text { Flexión (+) } \\
\text { /extensión (-) }\end{array}$ & $-15.1(5.9)$ & $-9.5(5.7)$ & $-7.0(6.2)$ & $-7.4(6.8)$ & $-12.2(5.7)$ & $-12.1(5.4)$ & $-10.9(5.7)$ \\
\hline $\begin{array}{l}\text { Aducción(+) } \\
\text { /abducción (-) }\end{array}$ & $-0.01(0.5)$ & $-0.07(0.5)$ & $0.03(0.4)$ & $-0.02(0.3)$ & $0.01(0.4)$ & $0.01(0.5)$ & $0.1(0.4)$ \\
\hline $\begin{array}{l}\text { Rotación interna }(+) \\
\text { /externa }(-)\end{array}$ & $0.1(0.6)$ & $-0.2(0.8)$ & $-0.04(0.5)$ & $0.1(0.8)$ & $0.1(1.7)$ & $0.6(2.5)$ & $0.7(2.5)$ \\
\hline \multicolumn{8}{|l|}{ Pelvis } \\
\hline $\begin{array}{l}\text { Flexión (+) } \\
\text { /extensión (-) }\end{array}$ & $12.4(4.5)$ & $13.7(4.6)$ & $14.4(4.7)$ & $15.3(4.7)$ & $12.8(4.6)$ & $13.1(4.3)$ & $13.3(4.5)$ \\
\hline $\begin{array}{l}\text { Aducción(+) } \\
\text { /abducción (-) }\end{array}$ & $-0.02(0.5)$ & $0.04(0.5)$ & $-0.07(0.5)$ & $-0.2(0.6)$ & $-0.01(0.4)$ & $-0.01(0.5)$ & $-0.1(0.5)$ \\
\hline $\begin{array}{l}\text { Rotación interna }(+) \\
\text { /externa }(-)\end{array}$ & $-0.5(1.6)$ & $-0.5(2.1)$ & $-0.2(1.3)$ & $-0.5(2.0)$ & $-0.2(1.4)$ & $-0.3(1.6)$ & $-0.3(1.2)$ \\
\hline \multicolumn{8}{|l|}{ Cadera } \\
\hline $\begin{array}{l}\text { Flexión (+) } \\
\text { /extensión (-) }\end{array}$ & $19.6(4.3)$ & $21.1(4.3)$ & $21.5(4.8)$ & $22.2(3.8)$ & $19.9(4.3)$ & $20.2(4.3)$ & $20.6(4.2)$ \\
\hline $\begin{array}{l}\text { Aducción(+) } \\
\text { /abducción (-) }\end{array}$ & $1.9(1.6)$ & $2.8(1.5)$ & $2.8(1.5)$ & $2.8(1.6)$ & $1.9(1.6)$ & $2.1(1.4)$ & $1.8(1.5)$ \\
\hline $\begin{array}{l}\text { Rotación interna }(+) \\
\text { /externa }(-)\end{array}$ & $1.0(5.9)$ & $-0.2(5.8)$ & $0.1(5.2)$ & $-0.4(5.4)$ & $0.7(5.8)$ & $0.5(6.1)$ & $0.6(5.8)$ \\
\hline \multicolumn{8}{|l|}{ Rodilla } \\
\hline $\begin{array}{l}\text { Flexión (+) } \\
\text { /extensión (-) }\end{array}$ & $25.1(3.8)$ & $25.4(4.3)$ & $25.3(4.9)$ & $25.1(3.7)$ & $25.2(3.8)$ & $25.1(4.3)$ & $25.4(4.0)$ \\
\hline $\begin{array}{l}\text { Aducción(+) } \\
\text { /abducción (-) }\end{array}$ & $-1.9(2.5)$ & $-1.8(2.7)$ & $-1.9(2.7)$ & $-1.9(2.5)$ & $-1.7(2.7)$ & $-1.9(2.6)$ & $-1.8(2.6)$ \\
\hline $\begin{array}{l}\text { Rotación interna }(+) \\
\text { /externa }(-)\end{array}$ & $-13.1(10.7)$ & $-13.1(10.5)$ & $-12.6(10.1)$ & $-12.5(10.1)$ & $-13.3(10.5)$ & $-13.4(10.6)$ & $-12.8(10.6)$ \\
\hline
\end{tabular}

Tabla 1. Plano sagital, frontal y transversal en cada una de las condiciones experimentales. Los datos son expresados como media (desviación estandar). 
que gracias a las ruedas del carro los escolares no tienen que soportar el peso sobre su propia espalda. El aumento de la flexión del tronco podría provocar un aumento de la presión de los discos intervertebrales según reflejan estudios previos $[27,28]$, y constituye además, uno de los factores identificados como predictores de dolor musculoesquelético en niños [29].

En el plano sagital, la cadera y la pelvis también mostraron un aumento de la flexión conforme la carga de la mochila aumentaba, tal y como se mostró en estudios previos [12,18], mientras que en el caso del carro, ningún tipo de adaptaciones significativas fueron obtenidas en comparación con la locomoción normal. Esta diferencia de resultados obtenidos con el uso de la mochila y el carro podría deberse a que la cadera es una articulación especialmente importante para la propulsión y el frenado cuando caminamos [18]. Por ello, un aumento del peso de la mochila produciría un requerimiento mayor de dicha articulación, mientras que con el uso del carro, al no tener que soportar esa carga sobre la espalda, las necesidades de propulsión y frenado no aumentan con respecto a la locomoción normal.

Con respecto a los resultados obtenidos en el plano frontal y transversal, la cadera mostró un aumento de los movimientos de aducción usando el carro con el $10 \%$ y $20 \%$ comparado con el uso de la mochila con esas mismas cargas, y también una disminución de los movimientos de rotación llevando la mochila con el 20\% BW comparado con la locomoción sin carga. En el caso del carro no se obtuvieron diferencias en ninguno de los movimientos frontales ni transversales de los segmentos analizados, a pesar de que supone una carga asimétrica.

La mayoría de literatura científica previa recomienda la mochila cuando las condiciones de carga están dentro de los límites establecidos (10-15\% BW), quedando el carro relegado a situaciones en las que los escolares transportan mucha cantidad de peso [1], o directamente se ha rechazado su uso [20]. En este estudio, el uso del carro ha provocado una menor adaptación postural que el uso de la mochila, y en los casos en los que ha sido ne- cesaria alguna adaptación, estas han sido menos significativas que las producidas usando la mochila. Además, los resultados obtenidos ponen de manifiesto que incluso cuando se transportan cargas más ligeras, como el 10\% y el 15\% BW, el carro escolar puede suponer una mejor opción que el uso de la mochila debido a que permite un comportamiento muy próximo a la locomoción sin carga, y necesita de muy pocas adaptaciones posturales.

Destacar que los resultados obtenidos con este estudio permiten considerar al carro escolar una buena opción para que sea usado por escolares de educación primaria siempre que su peso esté entre el 10 y el $20 \%$ BW. Habría que destacar algunas situaciones en las que el carro necesita ser objeto de estudio, como por ejemplo ante la presencia de obstáculos, como escaleras o superficies con desniveles, dónde la doble opción del carro [usarlo tirando o con las asas colgado sobre los hombros), permitiría que el escolar lo pudiera transportar como una mochila tradicional, hasta que se profundice en un mayor análisis del carro en estas situaciones. A pesar de estas limitaciones, el uso del carro proveería de una mayor ventaja en situaciones estáticas en bipedestación, que cargando la mochila en la espalda, donde el uso del carro evita que el peso de los materiales escolares recaiga sobre su espalda (por ejemplo mientras espera el autobús, permanece de pie en la fila o sale del colegio).

\section{Conclusión}

El uso del carro escolar requiere de menor número de adaptaciones posturales que el uso de la mochila usando la misma carga, y además, en las situaciones que lo requería, estas fueron de menor magnitud. Por tanto, el carro escolar podría ser también una opción para los escolares de Educación Primaria.

\section{Agradecimientos}

A todos los padres y escolares que han formado parte de este trabajo. El trabajo de Orantes-Gonzalez ha sido financiado por el Ministerio de Educación, Cultura y Deporte (FPU13/00162). 


\section{Referencias}

1. American Academy of Pediatrics. Back to School Tips; 2016. Disponible en: https://www.aap.org/. Citado el 15 de Diciembre de 2016.

2. American Occupational Therapy Association. 1, 2, 3's of Basic Backpack Wearing; 2014. Disponible en: http://www.aota.org. Citado el 15 de Diciembre de 2016.

3. American Physical Therapy Association. Backpack Safety - MoveForward; 2016. Disponible en: http://www.apta.com. Citado el 15 de Diciembre de 2016.

4. Al-Saleem SA, Ali A, Ali SI, Alshamrani AA, Almulhem AM. A Study of School Bag Weight and Back Pain among Primary School Children in Al-Ahsa, Saudi Arabia. Epidemiology 2016; 6:2161-2165.

5. Fadhil FH. Low back pain in schoolchildren: the role of school bag weight and carrying way. J Nat Sci Res 2013; 3.

6. Orantes-Gonzalez E, Heredia-Jimenez J. La mochila escolar en Educación Primaria: hábitos, dolor de espalda y calidad de vida. Actas XXXIX Congreso de la Sociedad Ibérica de Biomecánica y Biomateriales. León; 2016:55.

7. Pau M, Pau M. Postural sway modifications induced by backpack carriage in primary school children: a case study in Italy. Ergonomics 2010; 53: 872-881.

8. Bort N, Pitarch A. Carritos o mochilas en la edad escolar. Fisioterapia 2002; 24: 63-72.

9. Garrido MSF, González MJL, Muñoz MM, García FP, Espinosa MR, Mora VJ. Hábitos de transporte de las mochilas escolares y relación con el dolor de espalda en nuestro medio. Vox Paediatrica 2011; 18: 24-29.

10. Ortega FZ, Sanchez MF, García RF, Schyke CEJ, Morales LZ. Predictors of scoliosis in school-aged children. Gac Med Mex 2014; 150: 524-530.

11. Pau M, Leban B, Paderi M, Nussbaum MA. Characterization of Pulling Forces Exerted by Primary School Children While Carrying Trolley Bags. Proc Hum Factors Ergon Soc Annu Meet 2013;57:501-505.

12. Devroey C, Jonkers I, de Becker A, Lenaerts G, Spaepen A. Evaluation of the effect of backpack load and position during standing and walking using biomechanical, physiological and subjective measures. Ergonomics 2007; 50: 728-742.

13. Singh T, Koh M. Effects of backpack load position on spatiotemporal parameters and trunk forward lean. Gait Posture. 2009;29:49-53.

14. Yen SC, Ling W, Magill R, McDonough A, Gutierrez GM. Temporal relationship between trunk and thigh contributes to balance control in load carriage walking. Gait Posture. 2011;34:402-8.

15. Smith B, Ashton KM, Bohl D, Clark RC, Metheny JB, Klassen S. Influence of carrying a backpack on pelvic tilt, rotation, and obliquity in female college students. Gait Posture. 2006;23:263-7.

16. Hyung EJ, Lee HO, Kwon YJ. Influence of load and carrying method on gait, specifically pelvic movement. J Phys Ther Sci 2016;28:2059-62.

17. Orantes-Gonzalez E, Heredia-Jimenez J, Beneck GJ. Children require less gait kinematic adaptations to pull a trolley than to carry a backpack. Gait Posture 2017; 52:189-193.

18. Chow DHK, Kwok MLY, Au-Yang ACK, Holmes AD, Cheng JCY, Yao FYD, Wong MS. The effect of backpack load on the gait of normal adolescent girls. Ergonomics 2005; 48: 642-656.

19. Orantes-gonzalez E, Heredia-jimenez J. Pulling a school trolley with different loads: A kinematic analysis in children. Gait Posture 2016;49:203-4.

20. Schmidt J, Docherty S. Comparison of the posture of school children carrying backpacks versus pulling them on trolleys. Clin Chiropr 2010;13(4):253-60.

21. Heredia-Jimenez J, Orantes-Gonzalez E. Definición de un modelo de marcadores epidérmicos de tórax-pelvis para la evaluación de la locomoción con mochila. Actas del XXXIX Congreso de la Sociedad Ibérica de Biomecánica y Biomateriales. León; 2016: 49.

22. Orantes-Gonzalez E, Heredia-Jimenez J, SotoHermoso VM. The effect of school trolley load on spatiotemporal gait parameters of children.Gait Posture 2015;42: 390-93.

23. Hong Y, Li JX. Influence of load and carrying methods on gait phase and ground reactions in children's stair walking. Gait Posture 2005;22(1):63-8.

24. Hong YL, Cheung CK. Gait and posture responses to backpack load during level walking in children. Gait Posture 2003;17(1):28-33.

25. Yen S-C, Ling W, Magill R, Mcdonough A, Gutierrez GM. Temporal relationship between trunk and thigh contributes to balance control in load carriage walking. Gait Posture 2011;34:402-8.

26. Li JX, Hong Y. Age difference in trunk kinematics during walking with different backpack weights in 6- to 12-year-old children. Res Sport Med an Int J 2004;135-42.

27. Rohlmannt A, Claes LE, Bergmannt G, Graichen F, Neef P, Wilke HJ. Comparison of intradiscal pressures and spinal fixator loads for different body positions and exercises. Ergonomics 2001;44(8):781-94.

28. Wilke HJ, Neef P, Caimi M, Hoogland T, Claes LE. New in vivo measurements of pressures in the intervertebral disc in daily life. Spine 1999;24(8):755-62.

29. Grimmer K, Dansie B, Milanese S, Pirunsan U, Trott P. Adolescent standing postural response to backpack loads: a randomised controlled experimental study. BMC Musculoskelet Disord 2002;3:10. 